\title{
Association of plasma VEGF-A, soluble VEGFR-1 and VEGFR-2 levels and clinical response and survival in advanced colorectal cancer patients receiving bevacizumab with modified FOLFOX6
}

\author{
YOSHIKO AOYAGI, HISAE IINUMA, ATSUSHI HORIUCHI, RYU SHIMADA and TOSHIAKI WATANABE \\ Department of Surgery, Teikyo University School of Medicine, Tokyo 173-0003, Japan
}

Received October 16, 2009; Accepted December 11, 2009

DOI: $10.3892 /$ ol_00000045

\begin{abstract}
For individualized bevacizumab-based therapy, non-invasive biomarkers are necessary. This study assessed the predictive value of plasma vascular endothelial growth factor (VEGF)-A, soluble VEGF receptor (sVEGFR)-1 and sVEGFR-2 levels as biomarkers for clinical response and survival in advanced colorectal cancer (CRC) patients treated with bevacizumab and modified FOLFOX6 (mFOLFOX6). Forty-six unresectable advanced CRC patients and 20 healthy controls were included in this study. CRC patients were treated with bevacizumab and mFOLFOX6. Pretreatment plasma VEGF-A, sVEGFR-1 and sVEGFR-2 levels were measured using the multiplex immunoassay. Plasma VEGF-A, sVEGFR-1 and sVEGFR-2 levels were significantly higher in CRC patients than in the healthy subjects. The plasma sVEGFR-1 levels in the responder patients [complete response $(\mathrm{CR}) /$ partial response (PR)] and stable disease (SD) patients were significantly lower than those in the progressive disease (PD) patients (CR/PR vs. PD, $\mathrm{p}=0.025$; $\mathrm{SD}$ vs. $\mathrm{PD}, \mathrm{p}=0.032$ ), while the plasma VEGF-A and sVEGFR-2 levels did not show any significant differences between the two groups of patients. Patients with higher sVEGFR-1 levels showed a significantly poorer progression-free survival (PFS) and overall survival (OS) than those with lower VEGFR-1 levels. In contrast, VEGF-A and sVEGFR-2 did not show any significant relationship between PFS and OS according to the status of each level. In the multivariate Cox proportional hazard regression
\end{abstract}

Correspondence to: Dr Hisae Iinuma, Department of Surgery, Teikyo University School of Medicine, 2-11-1 Kaga, Itabashi-ku, Tokyo 173-0003, Japan

E-mail: iinuma@med.teikyo-u.ac.jp

Abbreviations: VEGF, vascular endothelial growth factor; sVEGFR-1, soluble vascular endothelial growth factor receptor-1; sVEGFR-2, soluble vascular endothelial growth factor receptor-2; CRC, colorectal cancer

Key words: vascular endothelial growth factor-A, vascular endothelial growth factor receptor-1, vascular endothelial growth factor receptor-2, bevacizumab, FOLFOX, colorectal cancer analysis, sVEGFR-1 levels showed a significant relationship between PFS and OS. These results suggest that plasma sVEGFR-1 levels have a predictive value for clinical response and survival in advanced CRC patients treated with bevacizumab and mFOLFOX6. Larger scale studies are needed to further validate our results.

\section{Introduction}

Bevacizumab is a humanized monoclonal antibody against vascular endothelial growth factor (VEGF) (1,2). In Japan, the use of bevacizumab alongside chemotherapy was approved in 2007 for the treatment of unresectable advanced CRC patients. Subsequently, clinical trials of bevacizumab have been undertaken in combination with chemotherapy, such as 5-fluorouracil (5-FU) and leucovorin (LV), 5-FU and LV (5-FU/LV) plus oxaliplatin (FOLFOX), 5-FU/LV plus ilinotecan (FOLFIRI) and capecitabine plus oxaliplatin (XELOX) (3,4). For tailored individualized therapy, many attempts have been made to identify predictive biomarkers to help select those patients that will benefit from targeted agents such as the association between the KRAS mutation status and survival outcomes in patients with metastatic CRC treated with cetuximab (5). For bevacizumab, however, no established predictive biomarkers have been identified which are associated with either treatment response or survival in patients with advanced CRC $(3,6)$.

VEGF and its receptors are essential for the neovascularization of cancer. Numerous studies have indicated that VEGF expression in tumor specimens is correlated with microvessel density, metastasis, tumor growth and poor prognosis in a variety of human solid cancer types including colorectal cancer (CRC) $(7,8)$. High preoperative serum or plasma VEGF concentrations may predict poor prognosis in patients with CRC $(9,10)$. However, the values of VEGF levels as biomarkers of anti-angiogenic therapy have yet to be established and require further evaluation (5,11-13). The VEGF family consists of related homodimeric glycoproteins, including VEGF-A (also called VEGF), -B, -C, -D and -E. It is known that VEGF-A binds to two types of cell membrane receptors: VEGF (VEGFR)-1 and VEGFR-2, located in the endothelium. Moreover, VEGF-A stimulates endothelial migration, proliferation, permeability and survival 
$(14,15)$. In addition to these receptors, circulating soluble forms of VEGFR-1 (sVEGFR-1) and VEGFR-2 (sVEGFR-2) have attracted attention as potential biomarkers of various malignancies. The sVEGFR-1 has been examined both as a potential surrogate marker for disease progression and/or as a potential inhibitor of tumor angiogenesis in colon, breast and renal cell carcinoma (16-21). However, the clinical significance of plasma sVEGFR-1 and sVEGFR-2 levels as biomarkers of anti-angiogenic therapy combined with chemotherapy have yet to be sufficiently investigated.

The present study aimed to evaluate the predictive value of plasma VEGF-A, sVEGFR-1 and sVEGFR-2 levels as biomarkers for clinical response and survival in unresectable advanced CRC patients treated with bevacizumab and modified FOLFOX 6 (mFOLFOX6) as a first-line therapy.

\section{Materials and methods}

Patients and study treatment blood samples. Forty-six unresectable advanced CRC patients (TNM stage IV) and 20 healthy controls were enrolled in this study. The patients were treated with bevacizumab and mFOLFOX6 as a first-line therapy between 2007 and 2009. Bevacizumab was administered at a dosage of $5 \mathrm{mg} / \mathrm{kg}$ on day 1 of every two-week period. The regimen of mFOLFOX6 was: oxaliplatin $85 \mathrm{mg} / \mathrm{m}^{2}$ on day 1 and $5-\mathrm{FU} / \mathrm{LV}$ (LV $200 \mathrm{mg} / \mathrm{m}^{2}$ on day 1 , 5 -FU $400 \mathrm{mg} / \mathrm{m}^{2}$ on day 1 and $5-\mathrm{FU} 2,400 \mathrm{mg} / \mathrm{m}^{2}$ continuous infusion on days 1 and 2). The median number of cycles of bevacizumab and mFOLFOX6 were 10. Patients were treated until disease progression, development of unacceptable toxicity or patient refusal. Peripheral blood was obtained from each patient before the treatment of bevacizumab combined with mFOLFOX6. Informed written consent was obtained from patients included in the study.

VEGFR-A, sVEGFR-1 and sVEGFR-2 measurements. Plasma samples were collected from the peripheral blood of each patient by centrifugation and stored at $-80^{\circ} \mathrm{C}$ until use. VEGF-A, sVEGFR-1 and sVEGFR-2 plasma levels were measured using the multiplex human immunoassay kit and the multiplex human soluble cytokine receptor panel kit (both from Millipore Co., MA, USA). Measurements were performed as follows: each 96-well filter plate was washed with $200 \mu$ l wash buffer, followed by filtration under a vacuum. The standard and control were added into appropriate wells, followed by $25 \mu \mathrm{l}$ assay buffer, $25 \mu \mathrm{l}$ samples and matrix solution. The bead mix was diluted in wash buffer, and $25 \mu \mathrm{l}$ of the mix were added to each well. The plates were maintained at $4^{\circ} \mathrm{C}$ overnight. The following day, the medium was vacuumfiltered, and $25 \mu \mathrm{l}$ detection antibody was added to each well. The plates were incubated for $1 \mathrm{~h}$ at room temperature (RT). Streptavidin-phycoerythin $(25 \mu \mathrm{l})$ was added to each well and incubated for $30 \mathrm{~min}$ at RT. The wells were washed twice with $200 \mu \mathrm{l}$ wash buffer, and $150 \mu \mathrm{l}$ sheath fluid was added. The plates were read on a Luminex 200 ${ }^{\mathrm{TM}}$ (Millipore Co.), and data were analyzed by xPONENT and Milliplex analyst software. The samples were examined in duplicate.

Assessment of efficacy. Tumor responses were assessed every 4-6 weeks using RECIST criteria and classified into four
Table I. Patient characteristics.

\begin{tabular}{|c|c|c|}
\hline \multirow[b]{2}{*}{$\begin{array}{l}\text { Characteristics of the } \\
\text { patients }(n=46)\end{array}$} & \multicolumn{2}{|c|}{ VEGF-A levels } \\
\hline & No. of patients & Percent \\
\hline Median age (range) & $63(49-77)$ & \\
\hline \multicolumn{3}{|l|}{ Gender } \\
\hline Male & 33 & 71.7 \\
\hline Female & 13 & 28.3 \\
\hline \multicolumn{3}{|l|}{ Primary sites } \\
\hline Colon & 32 & 69.6 \\
\hline Rectum & 14 & 30.4 \\
\hline \multicolumn{3}{|l|}{ Site of metastasis ${ }^{\mathrm{a}}$} \\
\hline Liver & 22 & 47.8 \\
\hline Lung & 17 & 34.1 \\
\hline Lymph node & 15 & 32.6 \\
\hline Omentum & 14 & 30.4 \\
\hline Local & 6 & 13.0 \\
\hline Bone & 1 & 2.2 \\
\hline \multicolumn{3}{|l|}{ Serum CEA } \\
\hline $\operatorname{CEA}(+)$ & 35 & 76.1 \\
\hline CEA (-) & 11 & 23.9 \\
\hline \multicolumn{3}{|l|}{ Serum CA19-9 } \\
\hline CA19-9 (+) & 16 & 34.8 \\
\hline CA19-9 (-) & 30 & 65.2 \\
\hline
\end{tabular}

averlapping cases are included.

groups: complete response (CR), partial response (PR), stable disease (SD) and progressive disease (PD). Response rates were calculated by the number of patients with $\mathrm{CR}$ or PR.

Statistical analysis. Plasma VEGF-A, sVEGFR-1 and sVEGFR-2 levels between the advanced CRC patients and healthy controls were analyzed by the Student's t-test. Differences in the markers between the clinical responses were examined with analysis of variance and multi-comparison tests. The correlation between VEGF-A, sVEGFR-1 and sVEGFR-2 and the clinicopathological parameters was evaluated using Fisher's exact and the Chi-square tests. Progression-free (PFS) and overall survival (OS) curves were analyzed using the Kaplan-Meier method, and the differences were examined using log-rank tests. Univariate and multivariate analyses were performed using Cox proportional hazard regression analysis. The tests were analyzed using JMP software (SAS Institute Inc., Cary, NC, USA). Statistical significance was determined from two-sided tests as $\mathrm{p}<0.05$.

\section{Results}

Patient characteristics. Table I shows the characteristics of the patients in this study. The median age was 63 years (range 49-77), with 33 male (71.7\%) and 13 female (28.3\%) patients. The patients had a good performance status (ECOG PSO). As 
Table II. VEGF-A, sVEGFR-1 and sVEGFR-2 levels of CRC patients and healthy controls.

\begin{tabular}{lrrcr}
\hline & No. & $\begin{array}{c}\text { Median } \\
(\mathrm{pg} / \mathrm{ml})\end{array}$ & $\begin{array}{c}\text { Average } \pm \text { SD } \\
(\mathrm{pg} / \mathrm{ml})\end{array}$ & P-value \\
\hline VEGF-A & & & & 0.004 \\
Controls & 20 & 54.4 & $68.9 \pm 69.2$ & \\
Patients & 46 & 158.0 & $194.0 \pm 178.6$ & \\
sVEGFR-1 & & & & 0.048 \\
Controls & 20 & 334.9 & $375.6 \pm 200.5$ & \\
Patients & 46 & 610.5 & $668.3 \pm 661.5$ & \\
sVEGFR-2 & & & & $<0.001$ \\
Controls & 20 & 10938.8 & $10665.7 \pm 3207.4$ & \\
Patients & 46 & 17800.5 & $17296.6 \pm 3987.0$ & \\
\hline
\end{tabular}

primary tumor sites, 32 patients $(69.6 \%)$ had colon cancer and 14 patients $(30.4 \%)$ had rectal cancer. The evaluable tumor sites for treatment were the liver (22 patients; 47.8\%), lung (17 patients; $34.1 \%$ ), lymph node (15 patients; $32.6 \%$ ), omentum (14 patients; 30.4\%), local (6 patients; 13.0\%) and/or bone metastasis (1 patient; 2.2\%). Serum CEA-positive patients numbered $35(76.1 \%)$, and there were 16 serum CA19-9positive patients $(34.8 \%)$.

Comparison of VEGF-A, sVEGFR-1 and SVEGFR-2 levels in CRC patients and healthy controls. Table II shows the comparison of plasma VEGF-A, sVEGFR-1 and sVEGFR-2 levels in patients with metastatic CRC and healthy controls. Plasma VEGF-A, sVEGFR-1 and sVEGFR-2 levels were
Table III. Plasma VEGF-A, sVEGR-1 and sVEGFR-2 levels and clinical response.

\begin{tabular}{lcrcc}
\hline & $\begin{array}{c}\text { No. of } \\
\text { patients }\end{array}$ & $\begin{array}{c}\text { Median } \\
(\mathrm{pg} / \mathrm{ml})\end{array}$ & $\begin{array}{c}\text { Average } \pm \text { SD } \\
(\mathrm{pg} / \mathrm{ml})\end{array}$ & P-value \\
\hline VEGF-A & & & & \\
CR/PR & $11^{\mathrm{a}}$ & 186.0 & $156.4 \pm 71.5$ & $0.300^{\mathrm{b}}$ \\
SD & 17 & 106.0 & $144.5 \pm 140.9$ & $0.073^{\mathrm{c}}$ \\
PD & 18 & 214.2 & $249.5 \pm 214.9$ & \\
sVEGFR-1 & & & & \\
CR/PR & $11^{\mathrm{a}}$ & 172.0 & $197.8 \pm 46.8$ & $0.025^{\mathrm{b}}$ \\
SD & 17 & 250.0 & $470.7 \pm 583.9$ & $0.032^{\mathrm{c}}$ \\
PD & 18 & 860.0 & $906.2 \pm 694.7$ & \\
sVEGFR-2 & & & & \\
CR/PR & $11^{\mathrm{a}}$ & 16010.0 & $16148.9 \pm 2273.4$ & $0.486^{\mathrm{b}}$ \\
SD & 17 & 17210.0 & $17276.8 \pm 5780.3$ & $0.587^{\mathrm{c}}$ \\
PD & 18 & 18035.5 & $17549.7 \pm 2570.2$ & \\
\hline
\end{tabular}

${ }^{\mathrm{a} C R}, 1 ; \mathrm{PR}, 10 .{ }^{\mathrm{b}} \mathrm{CR} / \mathrm{PR}$ vs. PD; ' $\mathrm{SD}$ vs. PD.

significantly higher in patients with metastatic CRC than in the healthy controls.

Plasma VEGF-A, sVEGFR-1 and sVEGFR-2 levels and clinical response. In this study, the patients received bevacizumab plus mFOLFOX6 as a first-line therapy. After treatment, 1 patient achieved CR, 10 patients had PR, 17 patients had SD and 18 patients had PD. The overall response rate was $23.9 \%$ (11/46). Table III shows the association between the plasma

Table IV. Association of patient characteristics and VEGF-A, sVEGFR-1 and sVEGFR-2 levels.

\begin{tabular}{|c|c|c|c|c|c|c|c|c|c|}
\hline \multirow[b]{2}{*}{$\begin{array}{l}\text { Characteristics } \\
\text { of the patients } \\
(n=46)\end{array}$} & \multicolumn{3}{|c|}{ VEGF-A levels } & \multicolumn{3}{|c|}{ sVEGFR-1 levels } & \multicolumn{3}{|c|}{ sVEGFR-2 levels } \\
\hline & $\begin{array}{l}\text { High } \\
(n=24)\end{array}$ & $\begin{array}{c}\text { Low } \\
(n=22)\end{array}$ & P-value & $\begin{array}{l}\text { High } \\
(n=23)\end{array}$ & $\begin{array}{c}\text { Low } \\
(n=23)\end{array}$ & P-value & $\begin{array}{l}\text { High } \\
(n=23)\end{array}$ & $\begin{array}{c}\text { Low } \\
(n=23)\end{array}$ & P-value \\
\hline $\begin{array}{l}\text { Median age } \\
\text { (range) }\end{array}$ & $\begin{array}{c}61.0 \\
(46-77)\end{array}$ & $\begin{array}{c}64.5 \\
(49-76)\end{array}$ & 0.411 & $\begin{array}{c}62.0 \\
(46-77)\end{array}$ & $\begin{array}{c}64.0 \\
(50-74)\end{array}$ & 0.622 & $\begin{array}{c}61.0 \\
(46-74)\end{array}$ & $\begin{array}{c}65.0 \\
(52-76)\end{array}$ & 0.163 \\
\hline Gender & & & & & & & & & \\
\hline $\begin{array}{l}\text { Male } \\
\text { Female }\end{array}$ & $\begin{array}{r}20(83.3) \\
4(16.7)\end{array}$ & $\begin{array}{r}14(63.6) \\
8(36.4)\end{array}$ & 0.129 & $\begin{array}{r}17(73.9) \\
6(26.1)\end{array}$ & $\begin{array}{r}17(73.9) \\
6(26.1)\end{array}$ & 1.000 & $\begin{array}{r}19(82.6) \\
4(17.4)\end{array}$ & $\begin{array}{r}15(65.2) \\
8(34.8)\end{array}$ & 0.179 \\
\hline $\begin{array}{l}\text { Primary sites } \\
\text { Colon } \\
\text { Rectum }\end{array}$ & $\begin{array}{r}19(79.2) \\
5(20.8)\end{array}$ & $\begin{array}{r}15(68.2) \\
7(31.8)\end{array}$ & 0.397 & $\begin{array}{r}19(82.6) \\
4(17.4)\end{array}$ & $\begin{array}{r}15(65.2) \\
8(34.8)\end{array}$ & 0.179 & $\begin{array}{r}19(82.6) \\
4(17.4)\end{array}$ & $\begin{array}{r}15(65.2) \\
8(34.8)\end{array}$ & 0.179 \\
\hline $\begin{array}{l}\text { Serum CEA } \\
\text { CEA (+) } \\
\text { CEA (-) }\end{array}$ & $\begin{array}{r}19(79.2) \\
5(20.8)\end{array}$ & $\begin{array}{r}16(72.7) \\
6(27.3)\end{array}$ & 0.609 & $\begin{array}{r}17(73.9) \\
6(26.1)\end{array}$ & $\begin{array}{r}18(78.3) \\
5(21.7)\end{array}$ & 0.730 & $\begin{array}{r}17(73.9) \\
6(26.1)\end{array}$ & $\begin{array}{r}18(78.3) \\
5(21.7)\end{array}$ & 0.730 \\
\hline $\begin{array}{c}\text { Serum CA19-9 } \\
\text { CA19-9 (+) } \\
\text { CA19-9 (-) }\end{array}$ & $\begin{array}{r}6(25.0) \\
18(75.0)\end{array}$ & $\begin{array}{l}10(45.5) \\
12(54.5)\end{array}$ & 0.146 & $\begin{array}{r}7(30.4) \\
16(69.6)\end{array}$ & $\begin{array}{r}9(39.1) \\
14(60.9)\end{array}$ & 0.536 & $\begin{array}{r}8(34.8) \\
15(65.2)\end{array}$ & $\begin{array}{r}8(34.8) \\
15(65.2)\end{array}$ & 1.000 \\
\hline
\end{tabular}


A

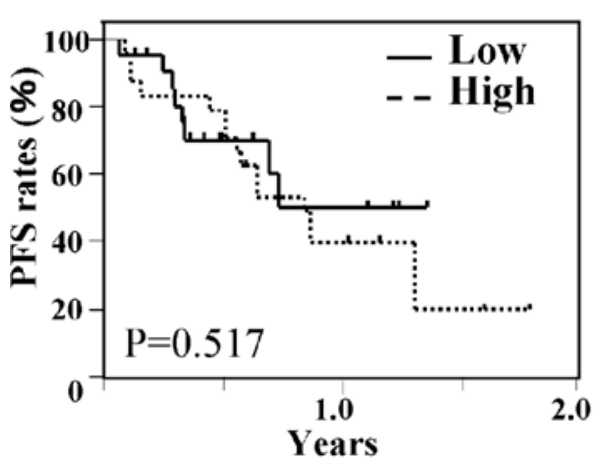

B

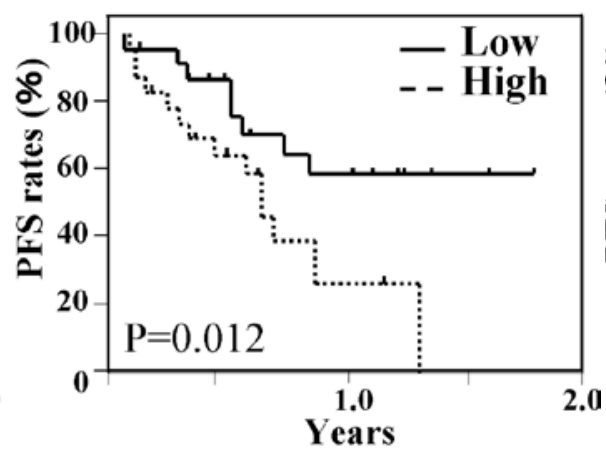

C SVEGFR-2

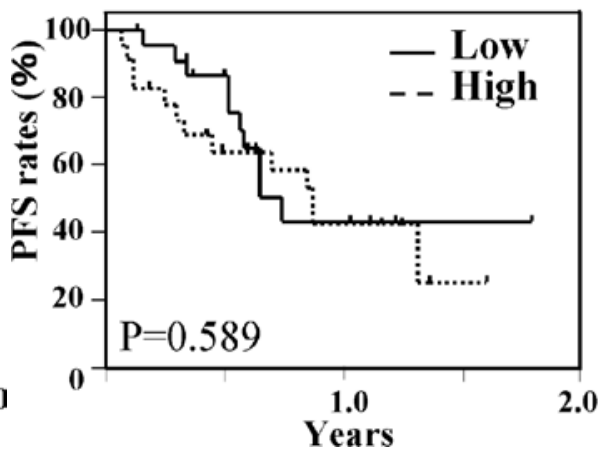

Figure 1. Progression-free survival curves according to the status of plasma VEGF-A, sVEGFR-1 and sVEGFR-2 levels. Kaplan-Meier progression-free survival (PFS) curves according to the status of plasma VEGF-A (A), sVEGFR-1 (B) and sVEGFR-2 (C) levels were examined. Each marker was divided into a higher- and lower-group by the medium level. (A) No significant differences were noted between patients with higher levels ( $\mathrm{n}=24)$ and those with lower levels $(\mathrm{n}=22)$. (B) There were significant differences between the sVEGFR-1 in the higher- $(\mathrm{n}=23)$ and lower-group $(\mathrm{n}=23)(\mathrm{P}=0.012)$. $(\mathrm{C})$ No significant differences were noted between the higher- $(n=23)$ and lower-group $(n=23)$.

A

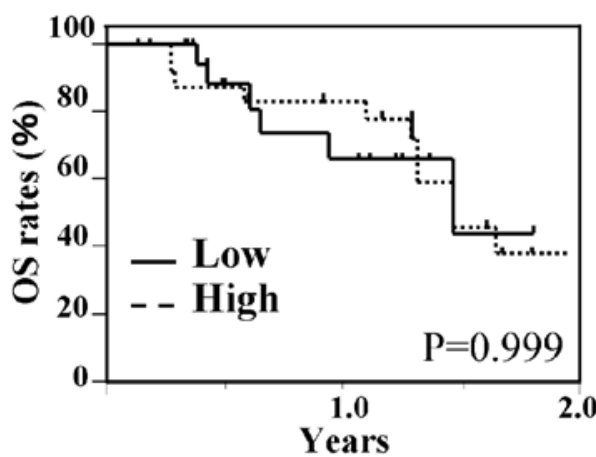

B

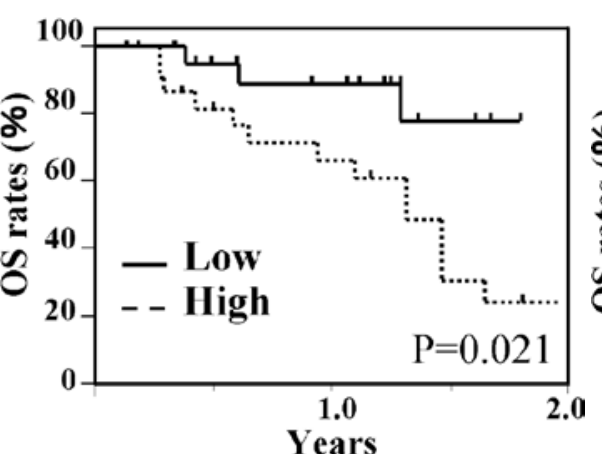

C

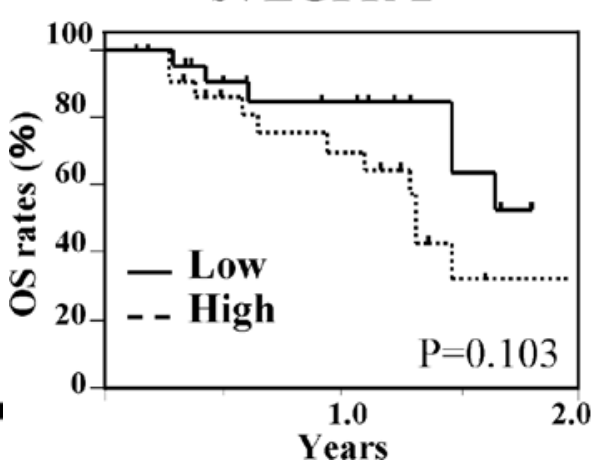

Figure 2. Overall survival curves according to the status of plasma VEGF-A, sVEGFR-1 and sVEGFR-2 levels. Kaplan-Meier overall survival (OS) curves according to the status of plasma VEGF-A (A), sVEGFR-1 (B) and sVEGFR-2 (C) levels were examined. Each marker was divided into a higher- and a lowergroup according to the medium level. (A) No significant differences were noted between the higher- $(\mathrm{n}=24)$ and lower-group ( $\mathrm{n}=22)$. (B) There were significant differences between sVEGFR-1 in the higher- $(n=23)$ and lower-group $(n=23)(P=0.021)$. (C) No significant differences were noted between the higher- $(n=23)$ and lower-group $(n=23)$.

VEGF-A, sVEGFR-1 and sVEGFR-2 levels and clinical responses. Regarding the VEGF-A levels, there were no significant differences between the responder, SD and PD groups. In contrast, the average level of sVEGFR-1 was $197.8 \pm 46.8 \mathrm{pg} / \mathrm{ml}$ in the responder patients, $470.7 \pm 583.9 \mathrm{pg} / \mathrm{ml}$ in the SD patients and $906.2 \pm 694.7 \mathrm{pg} / \mathrm{ml}$ in the PD patients. Significant differences were noted between the $C R / P R$ vs. PD group ( $p=0.025)$, and the SD vs. PD group ( $\mathrm{p}=0.032)$. No significant differences were noted when sVEGFR-2 levels and clinical responses were compared. These results suggest that sVEGFR-1 levels show a significant relationship with the clinical response.

Association of patient characteristics and VEGF-A, sVEGFR-1 and $s V E G F R-2$ levels. To examine the association of patient characteristics and VEGF-A, sVEGFR-1 and sVEGFR-2 levels, patients were divided into two groups (a higher level and a lower level group) by the setting of a cut-off based on median levels (Table IV). The median levels of VEGF-A, sVEGFR-1 and sVEGFR-2 were $165.0 \mathrm{pg} / \mathrm{ml}, 327.5 \mathrm{pg} / \mathrm{ml}$ and $17800.5 \mathrm{pg} / \mathrm{ml}$, respectively (data not shown). No statistically significant differences were noted in the patient characteristics and VEGF-A, sVEGFR-1 and sVEGFR-2 levels.

Plasma VEGF-A, sVEGFR-1 and sVEGFR-2 levels and survival. Fig. 1 shows the Kaplan-Meier PFS curves according to the status of plasma VEGF-A, sVEGFR-1 and sVEGFR-2 levels. In a comparative analysis based on the levels of sVEGFR-1, significant differences were noted between patients with higher and those with lower levels. In contrast, in the analysis of VEGF-A and sVEGR-2, no significant differences were found between patients with higher and those with lower levels. OS curves according to the status of plasma VEGF-A, sVEGFR-1 and sVEGFR-2 levels were then examined (Fig. 2). Patients with higher sVEGFR-1 levels showed a significantly poorer OS than those with lower VEGFR-1 levels. An analysis of VEGF-A and sVEGFR-2 showed no significant differences between patients with higher and those with lower levels of sVEGFR-1. These results suggest that sVEGFR-1 levels are significantly associated with the PFS and OS of patients treated with bevacizumab and mFOLFOX6. 
Table V. Multivariate analysis of biological factors for PFS.

\begin{tabular}{lccc}
\hline Factors & \multicolumn{3}{c}{ Multivariate analysis for PFS } \\
\cline { 2 - 4 } & $\begin{array}{c}\text { Regression } \\
\text { coefficient }\end{array}$ & $\begin{array}{c}\text { Hazard ratio } \\
(95 \% \text { CI })\end{array}$ & P-value \\
\hline VEGF-A & 0.364 & $1.439(0.505-4.303)$ & 0.496 \\
sVEGFR-1 & 1.119 & $3.063(1.189-8.520)$ & 0.021 \\
sVEGFR-2 & -0.143 & $0.866(0.343-2.184)$ & 0.758 \\
Serum CEA & -0.222 & $0.861(0.277-2.620)$ & 0.695 \\
Serum CA19-9 & 0.849 & $2.338(0.736-8.105)$ & 0.151 \\
\hline
\end{tabular}

PFS, progression-free survival; 95\% CI, 95\% confidence interval.

Multivariate analysis of biological factors for survival. Table V shows the multivariate analysis of biological factors for PFS. In this analysis, sVEGFR-1 levels showed a significant relationship with PFS. Table VI shows the multivariate analysis of biological factors for OS, and sVEGFR-1 levels showed a significant relationship with OS. In the analysis of clinicopathological factors of patients and survival, there was no significant relationship with PFS and OS (data not shown).

These results suggest that plasma sVEGFR-1 levels are a useful prognostic indicator for PFS and OS for advanced CRC patients treated with bevacizumab and mFOLFOX6.

\section{Discussion}

This study showed that plasma sVEGFR-1 levels, but not VEGF-A, and sVEGFR-2, are associated with clinical response and survival in advanced CRC patients treated with bevacizumab and mFOLFOX6.

It has been well established that VEGF is a key mediator of tumor vascularization. There is growing recognition of the central role that the VEGF family plays in angiogenesis, the formation of new blood vessels, which is necessary for the growth and spread of a tumor (22). The VEGF family consists of seven members, VEGF-A (also called VEGF), -B, -C, -D, $-\mathrm{E},-\mathrm{F}$ and placental growth factor (PIGF), which share eight cytokine residues in a VEGF homology domain (23). VEGF-A, particularly the VEGF165 and VEGF121 isoforms, plays an integral role in tumor angiogenesis both as an activator and survival factor in endothelial cells. Circulating plasma VEGF levels have been studied as a possible surrogate marker of angiogenesis in numerous malignancies (24). However, the applicability of plasma VEGF levels to predict the response and survival of patients treated with bevacizumab and chemotherapy has yet to be sufficiently proven. Burstein et al reported that lower levels of plasma VEGF were associated with longer time to progression in advanced breast cancer patients receiving bevacizumab and vinorelbine chemotherapy (25). In contrast, Denduluri et al reported that baseline levels of plasma VEGF in breast cancer patients did not predict clinical response to bevacizumab (12). In our study, pretreatment plasma VEGF-A levels in patients receiving
Table VI. Multivariate analysis of biological factors for OS.

\begin{tabular}{lccc}
\hline Factors & \multicolumn{3}{c}{ Multivariate analysis for OS } \\
\cline { 2 - 4 } & $\begin{array}{c}\text { Regression } \\
\text { coefficient }\end{array}$ & $\begin{array}{c}\text { Hazard ratio } \\
(95 \% \text { CI })\end{array}$ & P-value \\
\hline VEGF-A & -0.291 & $0.747(0.103-3.341)$ & 0.698 \\
sVEGFR-1 & 1.216 & $1.824(0.654-5.604)$ & 0.040 \\
sVEGFR-2 & -0.356 & $0.701(0.140-3.171)$ & 0.254 \\
Serum CEA & -0.165 & $0.848(0.229-4.018)$ & 0.816 \\
Serum CA19-9 & -0.356 & $0.701(0.140-3.171)$ & 0.647 \\
\hline
\end{tabular}

OS, overall survival; 95\% CI, 95\% confidence interval.

bevacizumab with mFOLFOX6 did not show any significant relationship between clinical response and survival. Notably, Holden et al reported that in their retrospective analysis of 398 metastatic CRC patients, the survival benefit associated with bevacizumab was independent of pretreatment plasma VEGF levels (26). Since VEGF is only one of the markers of antiangiogenesis, its significance in clinical response and survival may be asserted through several different pathways.

It is known that sVEGFR-1 and sVEGFR-2 are generated either via proteolytic cleavage of the ectodomain from the cell surface or via alternative mRNA splicing, which gives rise to a secreted polypeptide lacking a transmembrane region and functioning as a high-affinity receptor of VEGF. Expression of VEGFRs, including VEGFR-1 and VEGFR-2 (both of which are expressed in a number of tumor cell types in addition to endothelial cells), has been correlated with various disease stages (27-29). It has previously been shown that circulating levels of soluble forms of these receptors, which are not capable of signal transduction, bind VEGF in the bloodstream and reduce the levels of free VEGF. This limits the pro-angiogenic effects of VEGF at the endothelial cell level $(1,30)$. In particular, sVEGFR-1 has been studied, not only as a potential surrogate marker for disease progression, but also as a potential inhibitor of tumor angiogenesis in various types of cancers $(5,31)$. Previous studies have shown that sVEGFR-1 and/or sVEGFR-2 levels in plasma were higher in cancer patients than in healthy volunteers $(21,32,33)$. Our present data also demonstrated that plasma sVEGFR-1 and sVEGFR-2 levels of advanced CRC patients are significantly higher than those of healthy volunteers. Toi et al (18) and Yamaguchi et al (20) reported that sVEGFR-1 levels in tumor tissue were an independent prognostic indicator of disease progression in CRC patients. However, the predictive values of plasma sVEGFR-1 and sVEGFR-2 levels for chemotherapy responses are still controversial. Ustuner et al reported that no significant differences were detected between the concentration of serum sVEGFR-1 and sVEGR-2 and chemotherapy response in small cell lung cancer patients (34). In contrast, Wierzbowska et al reported correlations between the pretreatment plasma VEGFR-1 concentration, tumor burden and poor prognosis in acute myeloid leukemia (AML) patients (32). Additionally, the serum VEGFR-1/VEGF ratio had a greater prognostic value 
than VEGF alone in their study. Hu et al also reported that plasma sVEGFR-1, but not sVEGFR-2, was an independent prognostic factor in AML and myelodysplastic syndromes (21). Little is currently known regarding the potential of plasma sVEGFR-1 and sVEGFR-2 levels as a predictive biomarker for treatment response and survival in CRC patients treated with bevacizumab-based therapy. Our results in CRC patients indicated that the pretreatment level of sVGEFR-2 showed no association with clinical response and survival. This is in line with previous reports that failed to detect a predictive marker for bevacizumab with chemotherapy. Nevertheless, our data showed that plasma sVEGFR-1 levels predicted the treatment response and survival in advanced CRC patients treated with bevacizumab and mFOLFOX6. To the best of our knowledge, the present study is the first to suggest the predictive value of sVEGFR-1 for clinical response and survival in advanced CRC patients treated with bevacizumab and mFOLFOX6. It has been documented that the plasma sVEGFR-1 level is related to tumor phenotype or prognosis, suggesting that sVEGFR-1 has a significant biological function in tumor cells (30). Although it is difficult to elucidate the correlation between the plasma sVEGFR-1 levels and clinical response and survival, plasma sVEGFR-1 levels may reflect tumor malignancy and predict tumor progression in the metastatic site. Notably, Willett et al reported that pretreatment sVEGFR-1 levels in patients with rectal cancer were correlated with post-treatment tumor stage after combination therapy with bevacizumab, radiation and chemotherapy (11). These results support the possible predictive value of plasma sVEGFR-1 levels in CRC patients treated with bevacizumab-based chemotherapy. We await further studies that may elucidate, in detail, the association between plasma sVEGFR-1 and clinical response and survival.

Although there were limitations to the present study due to the small sample size and the fact that it was a single-arm study, we believe that our findings warrant the further evaluation of plasma sVEGFR-1 as a predictive marker for clinical response and survival in metastatic CRC patients. Larger scale studies are needed to further validate our results.

\section{Acknowledgements}

We thank Ms. J. Tamura for her excellent technical support. This work was supported by a Grant-in-Aid for Scientific Research (C) (21591734).

\section{References}

1. Hicklin DJ and Ellis LM: Role of the vascular endothelial growth factor pathway in tumor growth and angiogenesis. J Clin Oncol 23: 1011-1027, 2005.

2. Hurwits H, Fehrenbacher L, Novotny W, et al: Bevacizumab plus irinotecan, fluorouracil and leucovorin for metastatic colorectal cancer. N Engl J Med 350: 2335-2342, 2004.

3. Iwasaki $J$ and Nihira S: Anti-angiogenic therapy against gastrointestinal tract cancers. Jpn J Clin Oncol 16: 1-9, 2009.

4. Yamaguchi K, Boku N, Kato K, Komatsu Y, Muro K and Hamamoto Y: Preliminary efficacy, safety and operability of bevacizumab plus capecitabine plus oxaliplatin (XELOX) as first-line therapy in Japanese patients with initially unresectable metastatic colorectal cancer. Ann Oncol 19: viii130, 2008.

5. Karapetis CS, Khambata FS, Jonker DJ, et al: K-ras mutations and benefit from cetuximab in advanced colorectal cancer. $\mathrm{N}$ Engl J Med 359: 1757-1765, 2008.
6. Willett CG, Boucher Y, Duda DG, et al: Surrogate markers for antiangiogenic therapy and dose-limiting toxicities for bevacizumab with radiation and chemotherapy: continued experience of a Phase I trial and rectal cancer patients. J Clin Oncol 22: 8136-8139, 2005.

7. Galizia G, Lieto E, Ferraraccio F, et al: Determination of molecular marker expression can predict clinical outcome in colon carcinoma. Clin Cancer Res 10: 3490-3499, 2004.

8. Takahashi Y, Kitadai Y, Bucana CD, Cleary KR and Ellis LM: Expression of vascular endothelial growth factor and its receptor, KDR, correlates with vascularity metastasis and proliferation of human colon cancer. Cancer Res 55: 3964-3968, 1995.

9. Werther K, Christensen IJ and Nielsen HJ: Prognostic impact of matched preoperative plasma and serum VEGF in patients with primary colorectal carcinoma:Br J Cancer 86: 417-423, 2002.

10. Broll R, Erdmann H, Duchrow M, et al: Vascular endothelial growth factor (VEGF) - a valuable serum tumor marker in patients with colorectal cancer? Eur J Surg Oncol 27: 37-42, 2001.

11. Willett CG, Boucher Y, Duda DG, et al: Efficacy, safety and biomarkers of neoadjuvant bevacizumab, radiation therapy and fluorouracil in rectal cancer: a multidisciplinary phase II study. J Clin Oncol 18: 3020-3026, 2009.

12. Denduluri N, Yang SX, Berman AW, Nguyen D, Liewehr DJ, Steinberg SM and Swalin SM: Circulating biomarkers of bevacizumab activity in patients. Cancer Biol Ther 7: 15-20, 2008.

13. Ferrara $\mathrm{N}$ and Alitalo K: Clinical application of angiogenic growth factors and their inhibitors. Nat Med 5: 1359-1364, 1999.

14. Shibuya M: Structure and function of VEGF/VEGF-receptor system involved in angiogenesis. Cell Struct Func 26: 25-35, 2001.

15. Kendall RL and Thomas KA: Inhibition of vascular endothelial cell growth factor activity by an endogenously encoded soluble receptor. Proc Natl Acad Sci USA 90: 10705-10709, 1993.

16. Kendall RL, Wang $G$ and Thomas KA: Identification of a natural soluble form of the vascular endothelial growth factor receptor, FLT-1, and its heterodimerization with KDR. Biochem Biophys Res Commun 226: 324-328, 1996.

17. Harris AL, Reusch P, Barlenon B, Hang C, Dobbs $\mathrm{N}$ and Marme D: Soluble Tie2 and Flt1 extracellular domains in serum of patients with renal cancer and response to antiangiogenic therapy. Clin Cancer Res 7: 1992-1997, 2001.

18. Toi M, Bando H, Ogawa T, Muta M, Horing C and Weich HA: Significance of vascular endothelial growth factor (VEGF)/ soluble VEGF receptor-1 relationship in breast cancer. Int $\mathrm{J}$ Cancer 98: 14-18, 2002.

19. Tas F, Duranyildiz D, Oguz H, Camlica H, Yasasever V and Topuz E: Circulating serum levels of angiogenic factors and vascular endothelial growth factor receptors 1 and 2 in melanoma patients. Melanoma Res 16: 405-411, 2006.

20. Yamaguchi T, Nando H, Mori T, et al: Overexpression of soluble vascular endothelial growth factor receptor 1 in colorectal cancer: association with progression and prognosis. Cancer Sci 98: 405-410, 2007.

21. Hu Q, Dey AL, Yang Y, et al: Soluble vascular endothelial growth factor receptor 1 , and not receptor 2, is an independent prognostic factor in acute myeloid leukemia and myeloidysplastic syndromes. Cancer 100: 1884-1891, 2004.

22. Hormbrey E, Gillespie P, Turner K, Han C, Roberts A, McGrouther D and Harris AL: A critical review of vascular endothelial growth factor (VEGF) analysis in peripheral blood: is the current literature meaningful? Clin Exp Metastasis 19: 651-663, 2002.

23. Otrock ZK, Makarem JA and Shamseddine AI: Vascular endothelial growth factor family of ligands and receptors: review. Blood Cells Mol Dis 38: 258-268, 2007.

24. Poon RT, Fan ST and Wong J: Clinical implication of circulating angiogenic factors in cancer patients. J Clin Oncol 19: 1207-1225, 2001.

25. Burstein A, Chen Y-H and Parker LM: VEGF as a marker for outcome among advanced breast cancer patients receiving anti-VEGF therapy with bevacizumab and vinorelbine chemotherapy. Clin Cancer Res 14: 7871-7877, 2008.

26. Holden SN, Ryan E, Kearns A, Holmgren E and Hurwitz H: Benefit from bevacizumab (BV) is independent of pretreatment plasma vascular endothelial growth factor-A (pl-VEGF) in patients (pts) with metastatic colorectal cancer (mCRC). J Clin Oncol 123: 3555, 2005. 
27. Olsson AK, Dimberg A, Kreuger J and Claesson-Welsh L: VEGF receptor signaling - in control of vascular function. Nat Rev Mol Cell Biol 7: 359-371, 2006.

28. Herold MC, Steiner HH, Andl T, et al: Expression and functional significance of vascular endothelial growth factor receptors in human tumor cells. Lab Invest 79: 1573-1582, 1999.

29. Masood R, Cai J, Zheng T, Smith DL, Hinton DR and Gill PS: Vascular endothelial growth factor (VEGF) is an autocrine growth factor for VEGF receptor-positive human tumors. Blood 98: 1904-1913, 2001

30. Ferrara N, Gerber HP and LeCouter J: The biology of VEGF and its receptors. Nat Med 9: 669-676, 2003.

31. Holash J, Davis S, Papadopoulos N, et al: VEGF-Trap: a VEGF blocker with potent antitumor effects. Proc Natl Acad Sci USA 99: 11393-11398, 2003.
32. Wierzbowska A, Robak T, Wizesien-Kus A, Krawczynska A, Maranda EL and Urbanska-Rys H: Circulating VEGF and its soluble receptors sVEGFR-1 and sVEGFR-2 in patients with acute leukemia. Eur Cytokine Netw 14: 149-153, 2003.

33. AI-Moundhri M, Shukaili A, AI-Nabhani M, AI-Bahrani B, Burney IA, Rozovo A and Ganguly SS: Measurement of circulating levels of VEGF-A, $-\mathrm{C}$ and $-\mathrm{D}$ and their receptors, VEGFR- 1 and -2 in gastric adenocarcinoma. World Gastroenterol 14: 3879-3883, 2008

34. Ustuner Z, Saip P, Yasasever V, et al: Prognostic and predictive value of vascular endothelial growth factor and its soluble reseptor VEGFR-1 and VEGFR-2 levels in the sera of small cell lung cancer patients. Med Oncol 25: 394-399, 2008. 